\title{
Comparison of DNA Extraction Protocols and Molecular Targets to Diagnose Tuberculous Meningitis
}

\author{
Flavia Silva Palomo, ${ }^{1,2}$ Martha Gabriela Celle Rivero, ${ }^{1}$ \\ Milene Gonçalves Quiles, ${ }^{1}$ Fernando Pereira Pinto, ${ }^{2}$ \\ Antonia Maria de Oliveira Machado, ${ }^{2}$ and Antonio Carlos Campos Pignatari ${ }^{1}$ \\ ${ }^{1}$ Special Clinical Microbiology Laboratory (LEMC), Federal University of São Paulo (UNIFESP), São Paulo, SP, Brazil \\ ${ }^{2}$ Central Laboratory of São Paulo Hospital, Federal University of São Paulo (UNIFESP), São Paulo, SP, Brazil
}

Correspondence should be addressed to Flavia Silva Palomo; fla_bio@hotmail.com

Received 18 January 2017; Revised 22 March 2017; Accepted 27 April 2017; Published 30 May 2017

Academic Editor: Carlo Garzelli

Copyright (C) 2017 Flavia Silva Palomo et al. This is an open access article distributed under the Creative Commons Attribution License, which permits unrestricted use, distribution, and reproduction in any medium, provided the original work is properly cited.

\begin{abstract}
Tuberculous meningitis (TBM) is a severe form of extrapulmonary tuberculosis. The aims of this study were to evaluate in-house molecular diagnostic protocols of DNA extraction directly from CSF samples and the targets amplified by qPCR as an accurate and fast diagnosis of TBM. One hundred CSF samples from 68 patients suspected of TBM were studied. Four DNA extraction techniques (phenol-chloroform-thiocyanate guanidine, silica thiocyanate guanidine, resin, and resin with ethanol) were compared and CSF samples were used to determine the best target (IS6110, MPB64, and $h s p 65 \mathrm{KDa}$ ) by qPCR. The extraction protocol using the phenol-chloroform-thiocyanate guanidine showed the best results in terms of quantification and sensitivity of PCR amplification, presenting up to 10 times more DNA than the second best protocol, the silica guanidine thiocyanate. The target that showed the best result for TBM diagnosis was the IS6110. This target showed $91 \%$ sensitivity and $97 \%$ specificity when we analyzed the results by sample and showed $100 \%$ sensitivity and $98 \%$ specificity when we analyzed the results by patient. The DNA extraction with phenol-chloroform-thiocyanate guanidine followed by IS6110 target amplification has been shown to be suitable for diagnosis of TBM in our clinical setting.
\end{abstract}

\section{Background}

Tuberculosis is a serious infectious contagious disease that usually affects the lungs but can also affect other organs such as kidney, bone, and central nervous system (CNS) [1]. In 2011, cases of extra pulmonary tuberculosis in Brazil reached almost $16 \%$ of all cases of the disease [2] and about $6.3 \%$ of these (1.3\% of the total) were TBM [3].

The TBM is the most severe form of extra pulmonary tuberculosis that has a high morbidity and mortality [4, 5]. The definitive diagnosis of TBM depends on the $M$. tuberculosis agent detection from cerebrospinal fluid (CSF). Routinely, the survey of M. tuberculosis in CSF is carried out by conventional microbiological methods including ZiehlNeelsen smear, which has low sensitivity $(0-20 \%)$ and culture, which requires until 65 days to final result $[1,6]$.
The sensibility for detection of M. tuberculosis in CSF samples can be substantially increased from $70 \%$ to $100 \%$ and the time required to release laboratory results can be significantly decreased with the use molecular methods, including the polymerase chain reaction (PCR). The rapid identification of TBM through molecular analysis of CSF is an important factor for proper and early institution of antimicrobial treatment [7-9].

The aims of this study were to evaluate in-house molecular diagnostic protocols of DNA extraction directly from CSF samples and the targets amplified by qPCR as an accurate and fast diagnosis of TBM.

\section{Methods}

2.1. Clinical CSF Samples. We utilized the CSF samples sent for mycobacteria culture at the Central Laboratory of São 
TABLE 1: Real time PCR targets primers.

\begin{tabular}{lcccc}
\hline Target & Primer & Sequence $\left(5^{\prime}-3^{\prime}\right)$ & Product & Reference \\
\hline \multirow{2}{*}{ IS6110 } & $I S-F w$ & CGTGAGGGCATCGAGGTGGC & $245 \mathrm{bp}$ & {$[9]$} \\
& $I S-R v$ & CGCTAGGCGTCGGTGACAAA & & \\
Hsp $65 \mathrm{KDa}$ & $h s p 65-F w$ & GAGATCGAGCTGGAGGATCC & bp & \\
& $h s p 65-R v$ & AGCTGCAGCCCAAAGGTGTT & $240 \mathrm{bp}$ & {$[7]$} \\
MPB64 & MPB64-Fw & TCCGCTGCCAGTCGTCTTCC & & \\
& MPB64-Rv & GTCCTCGCAGTCTAGGCCA & & \\
\hline
\end{tabular}

Note. Fw: forward; Rv: reverse; bp: base pairs of nucleotidis.

Paulo Hospital, Federal University of São Paulo/UNIFESP, Brazil, in the period from January 2011 to June 2014. Aliquots were frozen at $-20^{\circ} \mathrm{C}$ and submitted to molecular tests after being thawed and centrifuged. These samples were also subjected to biochemical and cytological analysis and determination of adenosine deaminase (ADA) levels.

The samples were classified as true positive and negative after a survey in the database "BrazilianTBweb", other laboratory CSF data and evaluation of medical records.

2.2. Microbiological Diagnostic of CSF Samples. The microbial diagnosis of CSF samples was made in the Central Laboratory of São Paulo Hospital, Federal University of São Paulo/UNIFESP, Brazil. For each CSF a Ziehl-Neelsen smear and mycobacterium culture in Lowenstein Jensen solid medium were done.

2.3. DNA Extraction Protocols. Four extraction methods were analyzed: phenol-chloroform with guanidine thiocyanate $\left(\right.$ Brazol $^{\circledR}$, LabTrade, Brazil), silica-guanidine plus thiocyanate plus guanidine thiocyanate (QIAmp ${ }^{\circledR}$ DNA Mini Kit, Qiagen, USA), resin (Chelex ${ }^{\circledR} 100$ resin, BioRad, USA), and resin precipitated with ethanol.

Comparison of extraction was determined by a serial 10fold dilutions prepared in four different diluents (ultrapure water, turbid, xanthochromic, and hemorrhagic CSF pools known negative for TBM) from a 0.5 McFarland $(1.5 \times$ $10^{8} \mathrm{CFU} / \mathrm{mL}$ ) suspension of M. tuberculosis ATCC 25177.

The extracted DNA was measured and analyzed by NanoVue ND-100 (Thermo Fisher Scientific, Wilmington, USA) and the amplification sensitivity was determined by IS6110 target. The DNA extraction protocol presenting the best results was chosen for target and clinical samples analysis.

Phenol-Chloroform-Thiocyanate Guanidine (Brazol, LabTrade, Brazil) DNA Extraction. An aliquot of $200 \mu \mathrm{L}$ CSF sample sediment was added to a microtube containing $500 \mu \mathrm{L}$ of Brazol and mixture. Then ice-cold chloroform $(300 \mu \mathrm{L})$ was added. The mixture was homogenized and centrifuged at $12,000 \mathrm{rpm}$ for 15 minutes at $8^{\circ} \mathrm{C}$. The supernatant was removed and transferred to another microtube containing $500 \mu \mathrm{L}$ of cold absolute ethanol. The mixture was stirred for 3-5 seconds by vortexing and centrifuged at 12,000 rpm for 15 minutes at $8^{\circ} \mathrm{C}$. The supernatant was removed and the "pellet" was washed with $500 \mu \mathrm{L}$ of ice-cold ethanol and centrifuged at $12,000 \mathrm{rpm}$ for 15 minutes at $8^{\circ} \mathrm{C}$. The supernatant was carefully removed and discarded. The "pellet" was incubated at room temperature to dryness and then dissolved in $30 \mu \mathrm{L}$ of ultrapure water (Invitrogen Life Technologies, USA) and allowed to dissolve at $65^{\circ} \mathrm{C}$ for 30 minutes. The samples were frozen at $-20^{\circ} \mathrm{C}$ when not used immediately.

Silica-Guanidine Thiocyanate (QIAamp DNA Mini Kit, Qiagen, USA) DNA Extraction. The protocol recommended by the manufacturer was used with $200 \mu \mathrm{L}$ of the CSF sediment.

Resin (Resin Chelex100, BioRad, USA) DNA Extraction. A $200 \mu \mathrm{L}$ aliquot of the CSF sample pellet was placed in $300 \mu \mathrm{L}$ of a $10 \%$ solution Chelex. This mixture was homogenized in vortex for 10 seconds, centrifuged quickly to remove excess fluid from the cover, and incubated at $95^{\circ} \mathrm{C}$ for 30 minutes in a heat block. The tube was homogenized for $15 \mathrm{sec}$ and centrifuged for 5 minutes at $13000 \mathrm{rpm}$. The supernatant containing the extracted DNA was transferred to a new tube. When not used immediately, the sample was frozen at $-20^{\circ} \mathrm{C}$.

Resin Precipitated with Ethanol (Chelex100 Resin, BioRad, USA) DNA Extraction. A $200 \mu \mathrm{L}$ aliquot of the CSF pellet was placed in $300 \mu \mathrm{L}$ of $10 \%$ solution Chelex. This mixture was homogenized in vortex for 10 seconds and centrifuged quickly to remove excess fluid from the cover. This mixture was incubated at $95^{\circ} \mathrm{C}$ for 30 minutes in a heat block. The tube was homogenized for $15 \mathrm{sec}$ and centrifuged for 5 minutes at $13000 \mathrm{rpm}$. The supernatant containing the extracted DNA was transferred to a new tube and $500 \mu \mathrm{L}$ of cold absolute ethanol was added. The mixture was stirred for 3-5 seconds by vortexing and centrifuged at $12,000 \mathrm{rpm}$ for 15 minutes at $8^{\circ} \mathrm{C}$. The "pellet" was washed with $500 \mu \mathrm{L}$ of ice-cold ethanol and centrifuged at $12,000 \mathrm{rpm}$ for 15 minutes at $8^{\circ} \mathrm{C}$. The supernatant was carefully removed and discarded. The "pellet" was incubated at room temperature to dryness and then dissolved in $30 \mu \mathrm{L}$ of ultrapure water (Invitrogen Life Technologies, USA) and allowed to dissolve at $65^{\circ} \mathrm{C}$ for 30 minutes. The samples were frozen at $-20^{\circ} \mathrm{C}$ when not utilized immediately.

2.4. Targets and PCR Protocols. Three different targets were analyzed for $M$. tuberculosis detection by real time PCR: IS6110 gene, $65 \mathrm{kDa}$ Heat Shock Protein gene (hsp65 KDa), and the MPB64 protein encoding gene (MPB64) (Table 1). The reaction was performed on Rotor-Gene 5 plex/HRM 
TABLE 2: List of microorganisms used as negative control.

\begin{tabular}{lc}
\hline Microorganisms & Origin \\
\hline Acinetobacter baumannii & ATCC 19606 \\
Candida spp. & Known strain \\
Cryptococcus neoformans & Known strain \\
Enterococcus faecalis & ATCC 29212 \\
Enterococcus faecium & Known strain \\
Escherichia coli & ATCC 35218 \\
Haemophilus influenzae & Known strain \\
Haemophilus spp. & Known strain \\
Klebsiella pneumoniae & Known strain \\
Listeria monocytogenes & Known strain \\
Mycobacterium chelonae & Known strain \\
Mycobacterium abscessus & Known strain \\
Mycobacterium avium & Known strain \\
Mycobacterium gordonae & Known strain \\
Mycobacterium intracellulare & Known strain \\
Mycobacterium kansasii & Known strain \\
Mycobacterium lentiflavum & Known strain \\
Neisseria meningitidis & Known strain \\
Neisseria spp. & Known strain \\
Nocardia spp. & Known strain \\
Proteus mirabilis & ATCC 29245 \\
Pseudomonas aeruginosa & ATCC 27853 \\
Salmonella enterica & ATCC 14028 \\
Shigella flexneri & ATCC 12022 \\
Staphylococcus aureus & ATCC 29213 \\
Staphylococcus epidermidis & ATCC 12228 \\
Streptococcus agalactiae & ATCC 43867 \\
Streptococcus pneumoniae & ATCC 12386 \\
Streptococcus pyogenes & ATCC 49619 \\
ATCC:Ameccis \\
\hline
\end{tabular}

ATCC: American Type Collection Culture.

platform (Qiagen, USA) by melting analyses (QuantiFast SYBR Green PCR (Qiagen, USA)) on $20 \mu \mathrm{L}$ final volume. The limit of detection (LoD) was determined by a serial sevenfold dilutions prepared in ultrapure water (Sigma-Aldrich, Co. Ltd, Saint Louis, US).

The target specificity was carried out by testing the negative control group and CSF samples. The negative control group was made up by ATCC and known strains of bacteria, yeasts, and other mycobacteria (Table 2).

2.5. Real Time PCR Protocol. A reaction containing $10 \mu \mathrm{L}$ of QuantiFast SYBR Green PCR (Qiagen, USA), $0.5 \mathrm{mM}$ of each primer, and $2 \mu \mathrm{L}$ of sample was used for $M$. tuberculosis DNA detection directly from the CSF sample. Thermocycling was performed in the PCR system in the real time Rotor-Gene Q 5plex Platform (Qiagen, USA) using the following conditions: an initial cycle of five minutes at $95^{\circ} \mathrm{C}$, followed by 45 cycles of 30 seconds at $94^{\circ} \mathrm{C}$ and 45 seconds at the respective annealing temperature for IS6110 $\left(65^{\circ} \mathrm{C}\right)$, hsp $65 \mathrm{KDa}\left(62^{\circ} \mathrm{C}\right)$, and MPB64 $\left(61^{\circ} \mathrm{C}\right)$. At the conclusion of cycling a melting step ranging from $72^{\circ} \mathrm{C}$ to $95^{\circ} \mathrm{C}$ with an increase of $0.5^{\circ} \mathrm{C}$ per second was added for each gene.

2.6. Statistical Analysis. The results obtained were compared and evaluated clinically and analytically. For each test sensitivity $(S)$, specificity $(\mathrm{SP})$, positive predictive value (PPV), negative predictive value (NPV) and accuracy $(A)$ were calculated. The Kappa index $(k)$ was used to analyze the correlation between the tests. For analytical comparison, mycobacteria culture was considered as gold standard. For clinical analysis the patients and their respective samples were classified as true positive and true negative in accordance with the result of mycobacterium culture and clinical and laboratory data. Clinical data of patients were collected from electronic medical records and laboratory reports provided by the Central Laboratory of Hospital São Paulo and records in Brazilian TBweb (http://www.cvetb.saude.sp.gov.br/).

\section{Results}

Among 100 clinical CSF samples collected from 68 patients, 35 from 16 patients were considered true positive and 65 samples from 52 patients were true negative by clinical parameters.

The DNA extraction protocol using the phenol-chloroform-thiocyanate guanidine presented the highest final DNA concentration among four methods evaluated (Table 3 ).

From 35 clinical samples considered true positive by clinical parameters for TBM diagnosis, IS6110 PCR was able to detect 32 of them followed by culture and hsp65 KDa (16 samples) and MPB64 (12 samples). (Table 4).

Sixty-five samples were considered as true negative by clinical parameters for TBM diagnosis and culture was negative for all of them while 63 samples were negative by IS6110, MBP64, and $h s p 65 \mathrm{KDa}$ PCR. (Table 4).

From 16 patients considered true positive by clinical parameters for TBM diagnosis, IS6110 was able to correctly detect all of them followed by $h s p 65 \mathrm{KDa}$ (11 patients), culture (10 patients), and MBP64 (7 samples). (Table 5).

From 52 patients considered true negative by clinical parameters for TBM diagnosis, the culture was able to detect all of them as negative followed by IS6110 (51 patients), hsp65, and MBP64 (50 samples). (Table 5).

LoD, efficiency, Ct (cycle threshold) median and $T_{\mathrm{m}}$ (temperature of melting) median for IS6110, MPB 64, and hsp65 KDa are presented in Table 6. The primers were specific for $M$. tuberculosis and did not show cross reactivity against different microorganisms tested.

\section{Discussion}

Over the years several studies proposed and validated molecular techniques for diagnosis of TBM [8-14]. These studies considered the importance of developing a simple technique that was easily reproduced in laboratories with minimal resources $[10,11,13,15,16]$. The choice of the most appropriate extraction method and the target to be amplified are criteria 
TABLE 3: Nanovue results form four extraction protocols.

\begin{tabular}{|c|c|c|c|c|}
\hline Diluent & Extraction method & Sensitivity amplification & DNA $[\mathrm{ng} / \mu \mathrm{L}]$ & A260/280 \\
\hline \multirow{4}{*}{ Ultrapure water } & Phenol Chloroform & $10^{-10}$ & 24,5 & 1,53 \\
\hline & Silica & $10^{-9}$ & 2,3 & 1,18 \\
\hline & Resin & $10^{-7}$ & 1,2 & 0,93 \\
\hline & Resin ethanol & $10^{-4}$ & 0,3 & 0,36 \\
\hline \multirow{4}{*}{ Turbid CSF } & Phenol Chloroform & $10^{-10}$ & 28,5 & 1,59 \\
\hline & Silica & $10^{-10}$ & 22,2 & 1,45 \\
\hline & Resin & $10^{-7}$ & 19 & 1,08 \\
\hline & Resin ethanol & $10^{-3}$ & 0,1 & 0,01 \\
\hline \multirow{4}{*}{ Xanthochromic CSF } & Phenol Chloroform & $10^{-10}$ & 53 & 1,56 \\
\hline & Silica & $10^{-9}$ & 21 & 1,8 \\
\hline & Resin & $10^{-7}$ & 24,5 & 1,09 \\
\hline & Resin ethanol & $10^{-6}$ & 0,12 & 1,4 \\
\hline \multirow{4}{*}{ Hemorrhagic CSF } & Phenol Chloroform & $10^{-10}$ & 64,5 & 1,55 \\
\hline & Silica & $10^{-10}$ & 60,8 & 1,76 \\
\hline & Resin & $10^{-7}$ & 67 & 1,22 \\
\hline & Resin ethanol & $10^{-6}$ & 0,1 & 2,19 \\
\hline
\end{tabular}

TABLE 4: Culture and real time PCR results for 100 samples included on the study.

\begin{tabular}{lcccccc}
\hline & $S$ & SP & PPV & NPV & A & K \\
\hline Culture & $46 \%$ & $100 \%$ & $100 \%$ & $77 \%$ & $81 \%$ & 0,52 (Weak) \\
INS6110 & $91 \%$ & $97 \%$ & $94 \%$ & $95 \%$ & $75 \%$ & $0,89($ Strong) \\
MPB64 & $34 \%$ & $97 \%$ & $86 \%$ & $73 \%$ & $75 \%$ & $0,36(\mathrm{Minimal})$ \\
hsp65 KDa & $46 \%$ & $97 \%$ & $89 \%$ & $77 \%$ & $79 \%$ & $0,48($ Weak) \\
\hline
\end{tabular}

Note. S: sensitivity; SP: specificity; PPV: positive predictive value; NPV: negative predictive value; $A$ : accuracy; $k$ : Kappa index.

TABLE 5: Culture and real time PCR results for 100 samples included on the study.

\begin{tabular}{lcccccc}
\hline & $S$ & SP & PPV & NPV & A & K \\
\hline Culture & $63 \%$ & $100 \%$ & $100 \%$ & $90 \%$ & $91 \%$ & 9,72 (moderate) \\
INS6110 & $100 \%$ & $98 \%$ & $94 \%$ & $100 \%$ & $99 \%$ & 0,96 (almost perfect) \\
MPB64 & $44 \%$ & $96 \%$ & $78 \%$ & $85 \%$ & $84 \%$ & 0,47 (weak) \\
$h$ sp65 KDa & $69 \%$ & $96 \%$ & $85 \%$ & $91 \%$ & $90 \%$ & 0,69 (moderate) \\
\hline
\end{tabular}

Note. S: sensitivity; SP: specificity; PPV: positive predictive value; NPV: negative predictive value; $A$ : accuracy; $k$ : Kappa index.

TABLE 6: LoD, efficiency, Ct, and Tm results for real time PCR.

\begin{tabular}{lcccc}
\hline Target & LoD (CFU) & Efficiency & $\begin{array}{c}\text { Ct } \\
\text { Median }\end{array}$ & 31,09 \\
Median \\
\hline IS6110 & $10^{0}$ & 1,07 & 25,78 & 89,8 \\
Hsp 65 KDa & $10^{2}$ & 1,02 & 35,07 & 96,8 \\
MPB64 & $10^{3}$ & 1,07 & 30,7 \\
\hline
\end{tabular}

LoD: limit of detection; Ct: cycle threshold; $T_{\mathrm{m}}$ : temperature of melting.

that improve the precision, sensitivity and specificity of the PCR test [16-18].

The complexity of rich cell wall lipids of mycobacteria is a limiting factor for the success of some DNA extraction techniques [19-22], besides the fact that the microorganism is an intracellular pathogen, which can hinder the isolation of these microorganisms in clinical samples [19, 22]. Since the CSF samples from patients with TBM are usually paucibacillary it is recommended to recover the greatest amount of DNA as possible. 
The extraction protocol using the phenol-chloroformthiocyanate guanidine showed the best results in terms of quantification and sensitivity of PCR amplification, presenting up to 10 times more DNA than the second best protocol, the silica guanidine thiocyanate.

The techniques using phenol-chloroform, silica, and thiocyanate guanidine are described as excellent choices for DNA extraction in different biological materials, in addition to being inexpensive and simple [8-13].

The methodology of phenol-chloroform extraction and DNA purification utilized the Brazol, which has in its composition in addition to phenol, guanidine thiocyanate, a chaotropic agent that inactivates endonucleases and prevents DNA binding to other molecules and facilitates the separation of cellular debris [23-26]. Furthermore, the phenol which is a potent proteolytic agent, corrosive and caustic, contributes to lysis of the cell envelope of mycobacteria. In phenol extraction, solubilization and denaturation of proteins and lipids occur efficiently [26]. The chloroform used in this method is also a good protein denaturing detergent and a major solvent of fats, which probably contributed to the removal of the lipid layer of the mycobacteria cell wall. Regarding the biohazard risk of phenol-chloroform, all tests were done following the biosafety manuals and chemical waste disposal regulations of our country.

The target that showed the best amplification results was the IS6110 qPCR with a sensitivity of $100 \%$ and specificity of $79 \%$, when compared to culture. The sample analysis for IS6110 qPCR amplification showed 91\% sensitivity and 97\% specificity with the clinical diagnosis. When this analysis was grouped by patient, we showed a very good agreement with the clinical diagnosis with $100 \%$ sensitivity and $98 \%$ specificity.

These results can be explained since the gene encoding the MPB64 protein and the encoding gene heat shock protein (hsp65 KDa) have single copies in mycobacteria genome while the insertion gene IS6110 has multiple copies which makes the reaction more sensitive [7-9].

The IS6110 target sequence is a repetitive insertion of 1,350 base pairs present in M. tuberculosis complex species with different numbers of copies integrated into various chromosomal sites $[9,27,28]$. This molecular target promotes an increase in the sensitivity of the technique, which is an advantage over other targets. Only three patients had positive CSF samples for target IS6110 undiagnosed for TBM, but when we evaluated other laboratory criteria or clinical characteristics of these patients two of them had TBM and only one remained doubtful.

Commercial molecular tests for pulmonary tuberculosis diagnosis such as XpertMTB/RIF represent a significant advance, since it is automated and provides fast results. However, for TBM diagnosis its sensitivity is around $60 \%$ to $80 \%$. [29-31]. Other targets also can be used for M. tuberculosis molecular diagnosis, including the TRC4 primer a conserved repetitive element with specificity for $M$. tuberculosis complex [32].

There are few reports of M. tuberculosis lacking the IS6110 that eventually could be responsible for false negative results $[32,33]$; however, we considered that the benefit of a greater sensitivity of the IS6110 gene that can be found repeatedly in the genome M. tuberculosis justifies its use [11, 32, 33].

Thus, we believe we have demonstrated the feasibility of a molecular test for the diagnosis of TBM by an in-house real time PCR with analytical and clinical correlation to be used in laboratories with adequate cost benefit. Studies comparing other molecular methods of DNA extraction, other molecular targets, in-house protocols, and commercial platforms are warranted.

\section{Conclusion}

The combination of DNA extraction by phenol-chloroform and guanidine thiocyanate, (Brazol) and qPCR by IS6110 target amplification could be an effective tool for $M$. tuberculosis diagnosis directly from CSF samples.

\section{Conflicts of Interest}

The authors declare that they have no conflicts of interest.

\section{Acknowledgments}

This study was supported by a grant from Coordenação de Aperfeiçoamento de Pessoal de Nível Superior, Capes, Brazil.

\section{References}

[1] G. E. Thwaites, T. T. H. Chau, K. Stepniewska, and etal., "Diagnosis of adult tuberculous meningitis by use of clinical and laboratory features," The Lancet, vol. 360, no. 9342, pp. 1287$1292,2002$.

[2] T. C. de Oliveira, "Implementação de ações de vigilância epidemiológica em nível regional," Revista de Saúde Pública, vol. 23, no. 1, pp. 79-81, 1989.

[3] D. M. Mota, H. B. M. Beltrão, T. M. Lanzieri, L. C. Vieira, and M. Machado, "Avaliação econômica da rubéola e de estratégia de controle em situação de surto em Fortaleza (Ceará)," Saúde e Sociedade, vol. 20, no. 3, 2011.

[4] A. Cherian and S. V. Thomas, "Central nervous system tuberculosis," African Health Sciences, vol. 11, no. 1, 2011.

[5] D. H. Kennedy and R. J. Fallón, "Tuberculous meningitis," Journal of American Medical Association, vol. 241, no. 3, pp. 264268,1979 .

[6] D. Heemskerk, J. Day, T. T. H. Chau et al., "Intensified treatment with high dose rifampicin and levofloxacin compared to standard treatment for adult patients with tuberculous meningitis (tbm-it): protocol for a randomized controlled trial," Trials, vol. 12, no. 1, 2011.

[7] L. M. Wildner, C. L. Nogueira, da. Silva Souza, B. S. G. Senna, R. M. da Silva, and M. L. Bazzo, "Micobacterias: epidemiologia e diagnóstico," Revista De Patologia Tropical, vol. 40, no. 3, pp. 207-230, 2011.

[8] B. W. Lee, J. A. M. A. Tan, S. C. Wong et al., "DNA amplification by the polymerase chain reaction for the rapid diagnosis of tuberculous meningitis. Comparison of protocols involving three mycobacterial DNA sequences, IS6110, $65 \mathrm{kDa}$ antigen, and MPB64," Journal of the Neurological Sciences, vol. 123, no. 1, pp. 173-179, 1994. 
[9] A. W. Mir, A. Kirmani, R. Eachkoti, and M. A. Siddiqi, "Improved diagnosis of central nervous system tuberculosis by MPB64-target PCR," Brazilian Journal of Microbiology, vol. 39, no. 2, pp. 209-213, 2008.

[10] P. W. Hermans, D. Van Soolingen, J. W. Dale et al., "Insertion element IS986 from Mycobacterium tuberculosis: a useful tool for diagnosis and epidemiology of tuberculosis," Journal of Clinical Microbiology, vol. 28, no. 9, pp. 2051-2058, 1990.

[11] L. Chaidir, A. R. Ganiem, A. vander Zanden et al., "Comparison of real time IS6110-PCR, microscopy, and culture for diagnosis of tuberculous meningitis in a cohort of adult patients in indonesia," Plos ONE, vol. 7, no. 12, Article ID e52001, 2012.

[12] S. Haldar, N. Sharma, V. K. Gupta, and J. S. Tyagi, "Efficient diagnosis of tuberculous meningitis by detection of Mycobacterium tuberculosis DNA in cerebrospinal fluid filtrates using PCR," Journal of Medical Microbiology, vol. 58, no. 5, pp. 616624, 2009.

[13] F. C. D. Q. Mello and J. Fonseca-Costa, "A utilidade da biologia molecular no diagnóstico da," Jornal Brasileiro De Pneumologia, vol. 31, no. 3, 188 pages, 2005.

[14] T. Takahashi, M. Tamura, Y. Asami et al., "Novel wide-range quantitative nested real-time PCR assay for Mycobactenum tuberculosis DNA: clinical application for diagnosis of tuberculous meningitis," Journal of Clinical Microbiology, vol. 46, no. 5, pp. 1698-1707, 2008.

[15] T. Takahashi, M. Tamura, and T. Takasu, "The PCR-based diagnosis of central nervous system tuberculosis: up to date," Tuberculosis Research and Treatment, 2012.

[16] V. R. Bollela, D. N. Sato, and B. A. L. Fonseca, "Problems in the standardization of the polymerase chain reaction for the diagnosis of pulmonary tuberculosis," Revista de Saúde Pública, vol. 33, no. 3, pp. 281-286, 1999.

[17] C. M. Baratto and F. Megiolaro, "Comparação de diferentes protocolos de extração de dna de bactérias para utilização em RAPD-PCR," Ciência-ACET, vol. 3, no. 1, pp. 121-130, 2012.

[18] A. P. Adams, S. R. Bolin, A. E. Fine, C. A. Bolin, and J. B. Kaneene, "Comparison of PCR versus culture for detection of Mycobacterium bovis after experimental inoculation of various matrices held under environmental conditions for extended periods," Applied and Environmental Microbiology, vol. 79, no. 20, pp. 6501-6506, 2013.

[19] J. Sambrook and D. W. Russell, Molecular Cloning. A Laboratory Manual, Cold Spring Harbor Laboratory Press, New York, NY, USA, 3rd edition, 2001.

[20] M. J. Zumárraga, V. Meikle, A. Bernardelli et al., "Use of touchdown polymerase chain reaction to enhance the sensitivity of mycobacterium bovis detection," Journal of Veterinary Diagnostic Investigation, vol. 17, no. 3, pp. 232-238, 2005.

[21] Z.-Q. Zhang and M. Ishaque, "Evaluation of methods for isolation of DNA from slowly and rapidly growing mycobacteria," International Journal of Leprosy and Other Mycobacterial Diseases, vol. 65, no. 4, pp. 469-476, 1997.

[22] M. Jaber, A. Rattan, A. Verma, J. Tyagi, and R. Kumar, "A simple method of DNA extraction from mycobacterium tuberculosis," Tubercle and Lung Disease, vol. 76, no. 6, pp. 578-581, 1995.

[23] B. J. Wards, D. M. Collins, and G. W. de Lisle, "Detection of mycobacterium bovis in tissues by polymerase chain reaction," Veterinary Microbiology, vol. 43, no. 2, pp. 227-240, 1995.

[24] R. Boom, C. Sol, M. Beld, J. Weel, J. Goudsmit, and P. WertheimVan Dillen, "Improved silica-guanidiniumthiocyanate DNA isolation procedure based on selective binding of bovine alphacasein to silica particles," Journal of Clinical Microbiology, vol. 37, no. 3, pp. 615-619, 1999.

[25] I. N. De Almeida, W. Da Silva Carvalho, M. L. Rossetti, E. R. D. Costa, and S. S. De Miranda, "Evaluation of six different DNA extraction methods for detection of Mycobacterium tuberculosis by means of PCR-IS6110: preliminary study," BMC Research Notes, vol. 6, no. 1, Article 561, 2013.

[26] P. Chomczynski and N. Sacchi, "Single-step method of RNA isolation by acid guanidinium thiocyanate-phenol-chloroform extraction," Analytical Biochemistry, vol. 162, no. 1, pp. 156-159, 1987.

[27] A. S. Goldsborough and M. R. Bates, U.S. Patent Application No. 14/193, p. 680, 2014.

[28] D. Van Soolingen, P. W. Hermans, P. E. W. De Haas, D. R. Soll, and J. D. A. Van Embden, "Occurrence and stability of insertion sequences in Mycobacterium tuberculosis complex strains: evaluation of an insertion sequence-dependent dna polymorphism as a tool in the epidemiology of tuberculosis," Journal of Clinical Microbiology, vol. 29, no. 11, pp. 2578-2586, 1991.

[29] N. C. S. De Assis, M. L. Lopes, N. C. Cardoso, M. M. Da Costa, C. D. O. Sousa, and K. V. B. Lima, "Molecular diagnosis of pulmonary tuberculosis," Jornal Brasileiro De Patologia E Medicina Laboratorial, vol. 43, no. 1, pp. 1-7, 2007.

[30] N. T. Q. Nhu, D. Heemskerk, T. T. H. Chau et al., "Evaluation of GeneXpert MTB/RIF for diagnosis of tuberculous meningitis," Journal of Clinical Microbiology, vol. 52, no. 1, pp. 226-233, 2014.

[31] V. B. Patel, G. Theron, L. Lenders et al., "Diagnostic accuracy of quantitative PCR (Xpert MTB/RIF) for tuberculous meningitis in a high burden setting: a prospective study," PLoS Med, vol. 10, no. 10, Article ID e1001536, 2013.

[32] A. N. I. Sattar, S. K. Setu, A. A. Saleh, and S. Ahmed, "TRC4 gene based PCR assay in diagnosis of tuberculous meningitis," Journal of Medical Microbiology, vol. 8, no. 2, pp. 19-22, 2017.

[33] A. Berwal, K. Chawla, S. Vishwanath, and V. P. Shenoy, "Role of multiplex polymerase chain reaction in diagnosing tubercular meningitis," Journal of Laboratory Physicians, vol. 9, no. 2, pp. 145-147, 2017. 


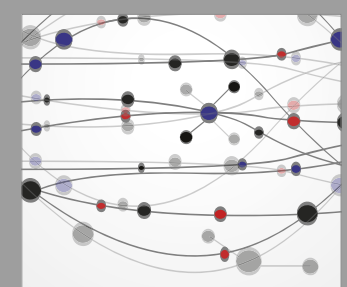

The Scientific World Journal
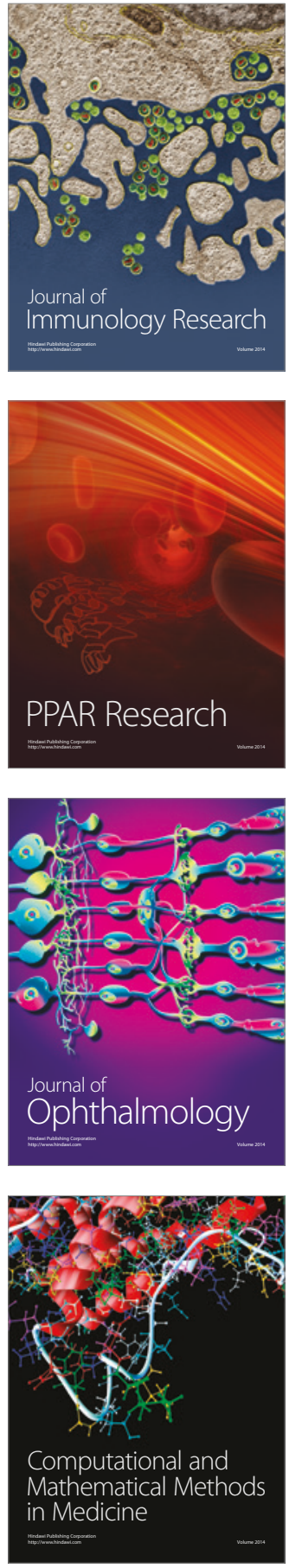

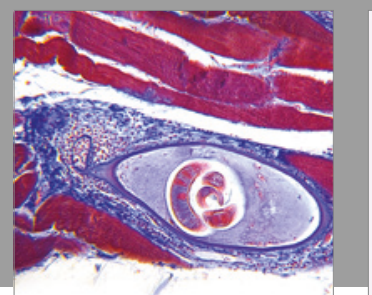

Gastroenterology Research and Practice
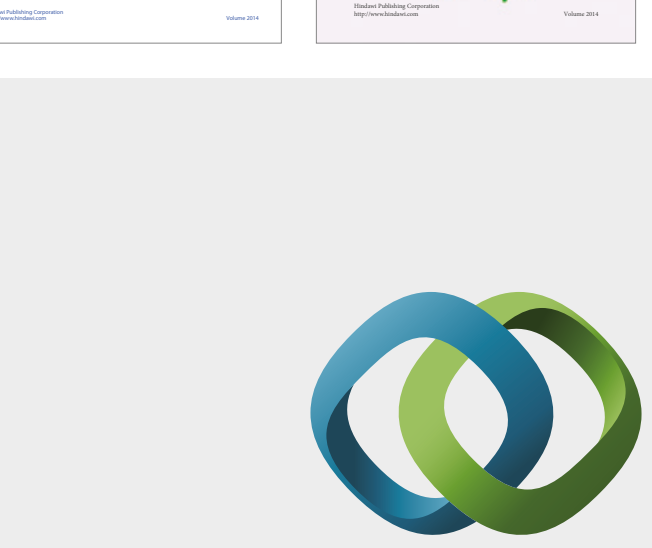

\section{Hindawi}

Submit your manuscripts at

https://www.hindawi.com
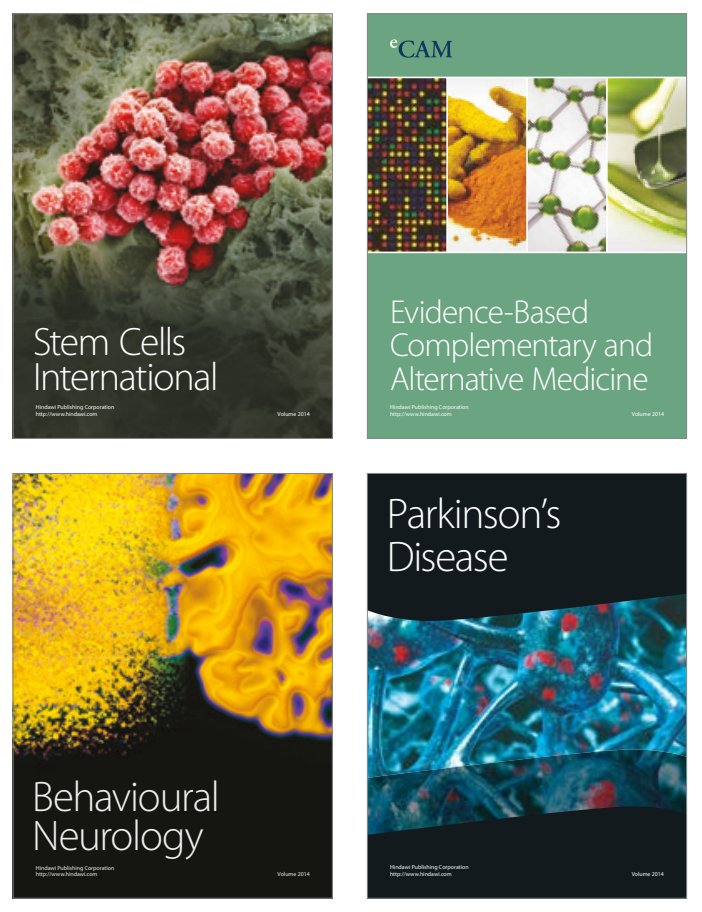
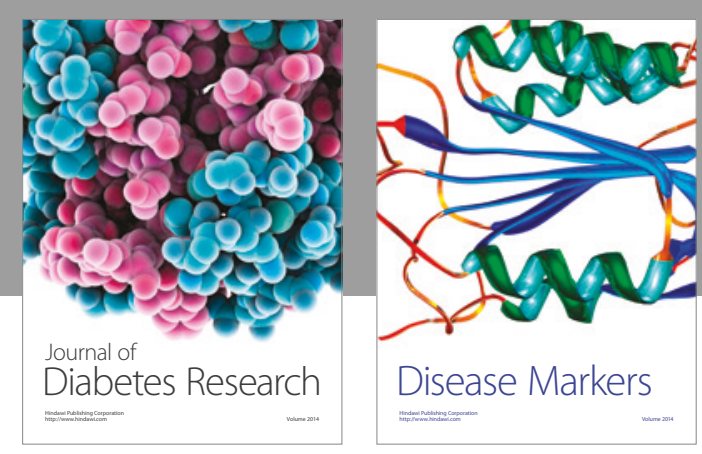

Disease Markers
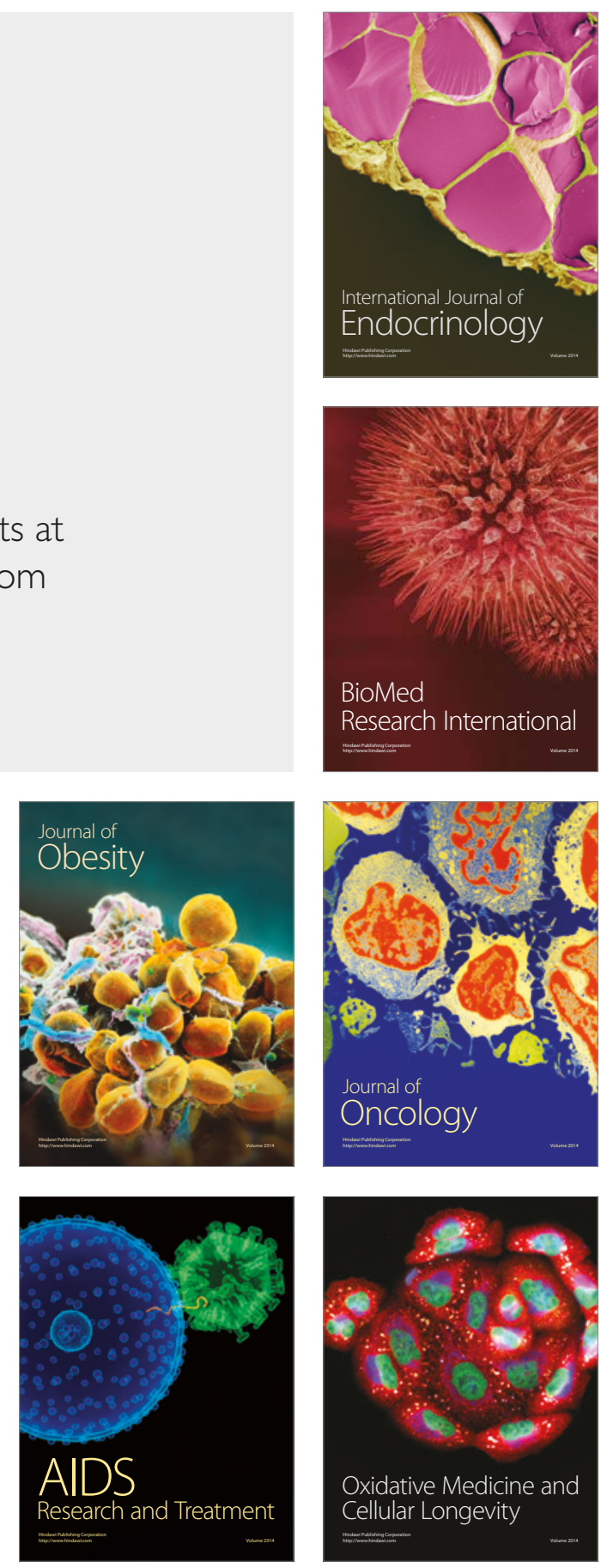